\title{
CONCERNING A CERTAIN SET OF ARRANGEMENTS
}

\section{BEN DUSHNIK}

1. Introduction, definitions, and notations. Let $m$ and $k$ be two positive integers, with $k \leqq m$. A set $S$ of arrangements (permutations) of the $m$ elements

$$
1,2, \cdots, m-1, m
$$

will be called $k$-suitable, if for every $k$-subset of the $m$ elements, say

$$
a_{1}, a_{2}, \cdots, a_{k}
$$

there exists an arrangement in $S$ in which $a_{k}$ follows all the $a_{i}$ with $i<k$. Such sets $S$ surely exist; for example, any set of $m$ arrangements whose terminal elements are $1,2, \cdots, m$, respectively, will obviously be $k$-suitable for any $k \leqq m$.

The smallest cardinal number $N$ such that there exists a set of $N$ arrangements which is $k$-suitable for $m$ will be denoted by $N(m, k)$; this is therefore a well defined positive integer for any $m$ and $k$, $k \leqq m$.

In any collection of arrangements of the first $m$ positive integers, the set of the terminal elements of the several arrangements will be called the end-elements, while all the other elements will then be referred to as mid-elements.

We shall hereafter refer to "suitable" sets of arrangements (omitting the numerical prefix) if the value of $k$ is clearly indicated by the context, or if the reference applies to all values of $k$.

In this paper we are primarily concerned with the evaluation of $N(m, k)$. The evaluation is not complete; the main result is given in Theorem I. In the last sections we use the results obtained to formulate an answer to a problem in connection with partially ordered sets.

Throughout this paper, $M$ will denote the set of the first $m$ positive integers. Small letters, insofar as they represent numbers, will represent positive integers.

\section{Some preliminary lemmas.}

LEMmA 1. If $1 \leqq k_{1} \leqq k_{2} \leqq m_{1} \leqq m_{2}$, then $N\left(m_{1}, k_{1}\right) \leqq N\left(m_{1}, k_{2}\right)$ $\leqq N\left(m_{2}, k_{2}\right)$.

LEMMA $2 . k \leqq N(m, k) \leqq m$.

Presented to the Society, September 5, 1947, under the title $A$ property of a set of permutations; received by the editors October 6, 1949 . 
Lemma 3. $N(m, 1)=1, N(m, 2)=2, N(m, m)=m$.

LEMma 4. There exists a suitable set of arrangements in which the terminal elements of the several arrangements are all distinct. Moreover, if the set of end-elements for this set of arrangements is denoted by

$$
a_{1}, a_{2}, \cdots, a_{s} \text {, }
$$

then the arrangement which terminates with $a_{i}(i=1,2, \cdots, s)$ begins with an (arbitrary) arrangement of the $a_{j}, j \neq i$.

The first three statements follow readily from the definitions. To prove Lemma 4, let

$$
A: A_{1}, A_{2}, A_{3}, \cdots, A_{\text {。 }}
$$

be a suitable set of $s$ arrangements. By Lemma 2, we may suppose that $s \leqq m$. Let $a_{1}$ be the terminal element of $A_{1}$, and let $A_{i}^{\prime}, i \neq 1$, be the arrangement obtainable from $A_{i}$ by transposing in it (if necessary) the element $a_{1}$ to the beginning, leaving the other elements undisturbed. It is easy to see that the set of arrangements

$$
A^{\prime}: A_{1}, A_{2}^{\prime}, A_{3}^{\prime}, \cdots, A_{\text {s }}^{\prime}
$$

is still suitable. Now let $a_{2}$ be the terminal element of $A_{2}^{\prime} ; a_{2}$ is clearly different from $a_{1}$. Let $A_{1}^{\prime \prime}, A_{3}^{\prime \prime}, A_{4}^{\prime \prime}, \cdots, A_{\mathrm{s}}{ }^{\prime}$ be the arrangements obtained from $A_{1}, A_{3}^{\prime}, A_{4}^{\prime}, \cdots, A_{3}^{\prime}$ by transposing in each one the element $a_{2}$ to the beginning, leaving the other elements undisturbed. Once again, the set of arrangements

$$
A^{\prime \prime}: A_{1}^{\prime \prime}, A_{2}^{\prime}, A_{3}^{\prime \prime}, \cdots, A_{2}^{\prime \prime}
$$

will remain suitable. Proceeding in the indicated manner, we shall finally get (after $s$ steps at most) a set of arrangements of the sort described in the lemma.

Lemma 5. For any natural $m$, the expression

$$
\frac{m}{x}+x-1
$$

is monotone decreasing as $x$ increases from 1 to $\mathrm{m}^{1 / 2}$.

To prove this, we need only to examine the graph of the hyperbola $x y=m+x^{2}-x$.

3. We shall now prove the following theorem.

ThEOREM I. For any integral $m \geqq 4$, let $j$ be any integer from 2 to 
$\left[m^{1 / 2}\right]$ inclusive, ${ }^{1}$ and let $k$ be any integer which satisfies the inequality (A): $\quad\left[\frac{m+j^{2}-j}{j}\right] \leqq k<\left[\frac{m+(j-1)^{2}-j+1}{j-1}\right]$.

Then $N(m, k)=m-j+1 .^{2}$

Proof. For a given integral $j$ between 2 and $\left[m^{1 / 2}\right]$ inclusive, the smallest value of $k$ defined by the inequality (A) is

$$
k_{1}=\left[\frac{m}{j}\right]+j-1,
$$

and the largest value is

$$
k_{2}=\left[\frac{m}{j-1}\right]+j-3
$$

(with the alternatives that either no value of $k$ is defined, or that $k_{2}=k_{1}$ ). Suppose now that we have already established the following facts:

(a) For $k_{2}$, one can construct a set of $m-j+1$ arrangements which is suitable; while

(b) no set of $m-j$ arrangements can be $k_{1}$-suitable.

Then, by (a) and (b),

$$
N\left(m, k_{2}\right) \leqq m-j+1, \quad N\left(m, k_{1}\right)>m-j,
$$

and the truth of our theorem will follow immediately from Lemma 1.

As to (a), consider $m-j+1$ arrangements which terminate respectively with the elements $1,2, \cdots, m-j, m-j+1$, and for which the arrangement which terminates with $s, 1 \leqq s \leqq m-j+1$, begins with an arbitrary arrangement of the other $m-j$ end-elements. The remaining elements $m-j+2, m-j+3, \cdots, m-1, m$, which constitute the set of mid-elements here, and whose number is clearly $j-1$, will therefore appear in each arrangement in the $j-1$ places immediately preceding the terminal element. The ordering of the mid-elements among themselves will be as follows. Separate the set of arrangements into $j-1$ mutually exclusive subsets, so that each subset contains either $[(m-j+1) /(j-1)]$ or $1+[(m-j+1) /(j-1)]$ arrangements, the separation being otherwise arbitrary. Now let the first subset have the element $m-j+2$ as the penultimate element in each of its arrangements, and let each of the remaining $j-2$ mid-

1 As usual, $[x]$ will denote the largest integer not exceeding $x$.

2 The case of $m=1,2$, or 3 or $j=1$ is taken care of by Lemma 3 . 
elements immediately precede the element $m-j+2$ in at least one arrangement of this subset. This is possible, since

$$
\begin{aligned}
{\left[\frac{m-j+1}{j-1}\right] } & =\left[\frac{m}{j-1}\right]-1 \geqq\left[\frac{m-1}{m^{1 / 2}-1}\right]-1 \\
& \geqq m^{1 / 2}-1 \geqq j-2 .
\end{aligned}
$$

We proceed similarly with the arrangements of each of the other subsets; thus, in the arrangements of the last subset the element $m$ will occupy the penultimate position, and for each of the other mid-elements there will be at least one arrangement of this subset in which this element will immediately precede $m$.

This set of $m-j+1$ arrangements is certainly $k_{2}$-suitable. Indeed, consider any $k_{2}$-subset of $M$, such as

$$
a_{1}, a_{2}, \cdots, a_{t}
$$

(where we put $k_{2}=t$ for typographical reasons). If $a_{t}$ if an end-element, the suitability is obvious. If $a_{t}$ is a mid-element, consider the subset of arrangements in which $a_{t}$ is the penultimate element, and suppose that not all of the end-elements of this subset are members of (C). In this case, again, there will surely be an arrangement wherein $a_{t}$ follows all the other $a$ 's. Finally, suppose that all the end-elements of the subset, in which $a_{t}$ is the penultimate element, belong to (C). The number of all other elements of (C) is thus at most

$$
\begin{aligned}
k_{2}-1-\left(\left[\frac{m}{j-1}\right]\right. & -1) \\
= & {\left[\frac{m}{j-1}\right]+j-3-1-\left[\frac{m}{j-1}\right]+1=j-3 . }
\end{aligned}
$$

Since the total number of subsets is $j-1$, it follows that there will be at least one subset such that neither the end-elements nor the penultimate element of the arrangements in this subset will be members of (C). Hence, an arrangement in this subset will exhibit $a_{t}$.as following all the other $a$ 's.

The proof of (a) is now complete.

Proof of (b). Suppose that the contrary is true: let $S$ be a set of $m-j$ arrangements of $M$ which is $k_{1}$-suitable. We suppose further that $S$ is of the sort described in Lemma 4 of $\$ 2$, so that the penultimate element of any arrangement in $S$ is a mid-element. Since the total number of mid-elements is $j$, there will be one of them, call it $a_{j}$, which occurs as the penultimate element in at most 


$$
t \equiv\left[\frac{m-j}{j}\right]
$$

arranguments of the set $S$. Denote the several end-elements of these arrangements by

$$
b_{1}, b_{2}, \cdots, b_{q}
$$

and let

$$
a_{1}, a_{2}, \cdots, a_{j-1}
$$

denote all the mid-elements other than $a_{j}$. Now

$$
q+j \leqq t+j=\left[\frac{m-j}{i}\right]+j=\left[\frac{m}{j}\right]+j-1=k_{1},
$$

so that

$$
\{c\} ; b_{1}, b_{2}, \cdots, b_{q} ; a_{1}, a_{2}, \cdots, a_{j}
$$

represents a $k_{1}$-subset $(\{c\}$ denotes the set of elements other than the $a$ 's and $b$ 's; it is empty if $t=q)$. But it is now clear that in any arrangement of $S$ where $a_{j}$ is not followed by a $b$, it will be followed by $a_{r}$, where $1 \leqq r<j$. Hence $S$ is not suitable, which is a contradiction to our original assumptions. Therefore (b) is true, and the proof of our theorem is now complete.

4. The theorem of the preceding section enables one to compute the value of $N(m, k)$ for any $k$ between

$$
\bar{m} \equiv\left[\frac{m}{\left[m^{1 / 2}\right]}\right]+\left[m^{1 / 2}\right]-1
$$

and $m$, inclusive. However, the method of proving statement (a) in the above section can be used to show that, if $k<\bar{m}$, then

$$
N(m, k) \leqq m-\left[m^{1 / 2}\right] \text {. }
$$

In particular, if $m=n^{2}+n$ or $n^{2}+2 n$, so that $\bar{m}=2 n$ or $2 n+1$, respectively, then

$$
N\left(n^{2}+n, 2 n-1\right) \leqq n^{2}, \quad N\left(n^{2}+2 n, 2 n\right) \leqq n^{2}+n .
$$

Direct computation by means of the theorem will give

$$
N\left(n^{2}+n-1,2 n-1\right)=n^{2}, \quad N\left(n^{2}+2 n-1,2 n\right)=n^{2}+n .
$$

Taking into account Lemma 1 of $\$ 2$, (I) and (II) together show the correctness of the following theorem, which is a rather slight extension of the previous one. 
THEOREM II. If $m=n^{2}+\alpha n, \alpha=1$ or 2 , then

$$
N(m-1,2 n+\alpha-2)=N(m, 2 n+\alpha-2)=m-n .
$$

5. An application. The "dimension" of a partially ordered set has been considered in a paper by Miller and the author ${ }^{3}$ since this term was used in a sense different from that employed by others, ${ }^{4}$ we give its definition here.

If $P$ and $L$ are, respectively, a partial ordering and a (simple) linear ordering of a set $S$, then $L$ will be called a linear extension of $P$ whenever the following is true: if $x$ and $y$ are elements of $S$ such that $x<y$ in $P$, then $x<y$ in $L$. For a given $P$ (on $S$ ), let $K$ be a collection of linear extensions of $P$ which has the property that, if $x$ and $y$ are elements of $S$ which are not ordered in $P$, then there exist in $K$ two extensions, $L^{\prime}$ and $L^{\prime \prime}$, such that $x<y$ in $L^{\prime}$ and $y<x$ in $L^{\prime \prime}$. Such a collection $K$ will be said to "realize" the partial order $P$. By the dimension of a partial order $P$, denoted by $\operatorname{dim} P$, we shall mean the smallest cardinal number $n$ such that $P$ is realized by a collection of $n$ extensions of itself.

It is known ${ }^{6}$ that every $P$ has a dimension, which is a finite number if the set on which $P$ is defined is finite. The dimension of certain special partial orders has been computed. For example, the partial order defined on the family of all subsets of a set of power $n$, with set inclusion as the ordering relation, has dimension $n .^{6}$ Similarly, consider the family of all $r$-subsets and $s$-subsets of $M$, where $1 \leqq r<s<m$; let $P(m, r, s)$ denote the partial order defined on this family by using, as above, set inclusion as the ordering relation. It has been shown ${ }^{7}$ that

$$
\operatorname{dim} P(m, 1, m-1)=m .
$$

In terms of the results obtained in this paper, the following generalization of (a) may now be stated.

THEOREM III. If $1<k<m$, then

$$
\operatorname{dim} P(m, 1, k)=N(m, k+1) \text {. }
$$

Proof. For convenience, put $P(m, 1, k)=P, \operatorname{dim} P=s, N(m, k+1)$ $=N$, and let

$$
L \equiv\left\{L_{1}, L_{2}, \cdots, L_{s}\right\}
$$

${ }^{3}$ See [1], in bibliography at the end.

- For example, by G. Birkhoff in his book Lattice theory.

- See [1, p. 601].

- See [3, p. 509].

7 See [1, p. 604]. 
be a set of $s$ linear extensions of $P$ which realize $P$. For any $i$ between 1 and $s$ inclusive, define $A_{i}$ as the arrangement of $M$ which results when, in $L_{i}$, we discard all the symbols which represent $k$-subsets, and replace all the other symbols (which represent 1-subsets) by their corresponding integers. The set

$$
A \equiv\left\{A_{1}, A_{2}, \cdots, A_{8}\right\}
$$

is thus a set of $s$ arrangements of the elements of the set $M$. Now, let

$$
a_{1}, a_{2}, \cdots, a_{k}, a_{k+1}
$$

be any $(k+1)$-subset of $M$. The $k$-subset $x \equiv\left(a_{1}, a_{2}, \cdots, a_{k}\right)$ and the 1 -subset $y \equiv\left(a_{k+1}\right)$ are not ordered in $P$; hence, in one of the extensions of $L$, we must have $x<y$, say

$$
x<y \text { in } L_{i} \text {. }
$$

Since $L_{i}$ is an extension of $P$, we must also have

$$
\left(a_{j}\right)<x \text { in } L_{i}, \quad j=1,2, \cdots, k \text {, }
$$

and therefore

$$
\left(a_{j}\right)<\left(a_{k+1}\right) \text { in } L_{i}, \quad 1 \leqq j \leqq k .
$$

It now follows, from the definition of $A_{i}$, that $a_{k+1}$ follows $a_{j}$ in $A_{i}$, $1 \leqq j \leqq k$. In other words, the set $A$ is $(k+1)$-suitable, and, since the number of arrangements in $A$ is $s$, we have

$$
N(m, k+1) \leqq s=\operatorname{dim} P(m, 1, k) .
$$

To complete the proof of our theorem, it suffices to show that

$$
\operatorname{dim} P(m, 1, k) \leqq N(m, k+1) .
$$

For this purpose, let

$$
A \equiv\left\{A_{1}, A_{2}, \cdots, A_{N}\right\}
$$

be a $(k+1)$-suitable set of $N$ arrangements of the elements of $M$. Transform any one of these arrangements, say

$$
A_{i}: a_{1}, a_{2}, \cdots, a_{m},
$$

into a linear ordering of 1 -subsets and $k$-subsets of $M$ in the following manner. The symbols $a_{r}$ will be re-interpreted to represent the corresponding 1-subsets of $M$, and these $a$ 's will be ordered according to the order of their appearance in $A_{i}$ (from left to right). If $x$ represents a $k$-subset of $M$, we insert $x$ into $A_{i}$ so that $x$ follows immediately after the last symbol which represents an element of $x$. If $x, y, \cdots, z$ 
are all the $k$-subsets each of which is "entitled" to the same position, then they are inserted as a unit in that position, the order among them being arbitrary. [To illustrate, if the transformed $A_{i}$ is denoted by $L_{i}$, then

$$
L_{i}: \quad a_{1}<a_{2}<\cdots<a_{k}<w<a_{k+1}<x<y<\cdots<z<\cdots
$$

where $w$ is the $k$-subset whose elements are $a_{1}, a_{2}, \cdots, a_{k}$, and $x, y, \cdots, z$, the several $k$-subsets each of which contains $a_{k+1}$ and some $k-1$ of the first $k$ elements of $A_{i}$.] It is obvious that the $L_{i}$ so obtained by transforming $A_{i}$ is a linear extension of $P$, so that

$$
L \equiv\left\{L_{1}, L_{2}, \cdots, L_{N}\right\}
$$

is a set of extensions of $P$.

Now, let $x$ and $y$ be any two subsets of $M$ which are not compared in $P$. There are three possibilities:

(a) $x$ and $y$ are both $k$-subsets;

(b) $x$ and $y$ are both 1 -subsets;

(c) $x$ is a $k$-subset, and $y$ is a 1 -subset which is not contained in $x$.

We discuss in detail only case (a), which is perhaps the most difficult. Let $r$ and $s$ be elements of $M$ such that $r$ is an element of $x$ which does not belong to $y$, and $s$ is an element of $y$ which does not belong to $x$. The union of $r$ and all the elements of $y$ is a $(k+1)$-subset, and since $A$ is $(k+1)$-suitable, there will exist $A_{i}$ in $A$ in which $r$ will follow all the elements of $y$. From the manner of construction of $L_{i}$, it clearly follows that

$$
y<x \text { in } L_{i} \text {. }
$$

Similarly, there will exist $L_{j}$ in $L, j \neq i$, such that $x<y$ in $L_{j}$. Cases (b) and (c) can be handled in analogous fashion, with the same final result. But all this means that $L$ realizes $P$. Since the number of extensions in $L$ is $N$, we have

$$
\operatorname{dim} P(m, 1, k) \leqq N=N(m, k+1) .
$$

As already pointed out, this completes the proof of Theorem III.

6. The partial ordering $P^{\prime}$ (of a set $S^{\prime}$ ) is said to be isomorphic to $P$ (on $S$ ) if there exists a 1-1 mapping of $S$ onto $S^{\prime}$ which preserves the partial ordering; $P^{\prime}$ is said to be dual to $P$ if the 1-1 mapping inverts the partial ordering, that is, if $x$ and $y$ are elements of $S$ whose images in $S^{\prime}$ are $x^{\prime}$ and $y^{\prime}$, then $x^{\prime}<y^{\prime}$ in $P^{\prime}$ if, and only if, $y<x$ in $P$. It is fairly obvious that 


$$
\operatorname{dim} P=\operatorname{dim} P^{\prime}
$$

whenever $P^{\prime}$ is either isomorphic with or dual to $P$.

Suppose now we have $1 \leqq r<s<m$. Every $r$-subset $x$ of $M$ determines uniquely an $(m-r)$-subset of $M$, namely, its complement $X^{\prime} \subseteq M-x$. Likewise, if $y$ is an $s$-subset of $M$, then $y^{\prime} \equiv M-y$ is an $(m-s)$-subset of $M$, and $y^{\prime} \subset x^{\prime}$ if, and only if, $x \subset y$. In other words, there is a "natural" 1-1 mapping of the family of all $r$-and $s$-subsets of $M$ onto the family of all $(m-r)$ - and $(m-s)$-subsets of $M$, and in terms of this mapping we see that $P(m, r, s)$ is dual to $P(m, m-s$, $m-r)$. Because of (a), the following theorem has thus been demonstrated:

THEOREM IV. If $1 \leqq r<s<m$, then

$$
\operatorname{dim} P(m, r, s)=\operatorname{dim} P(m, m-s, m-r) .
$$

Finally, we may state the following theorem:

TheOREM V. If $1<k<m$, then

$$
\operatorname{dim} P(m, m-k, m-1)=N(m, k+1) .
$$

This follows from the preceding theorem with $r=1$, if we also take Theorem III into account.

7. Conclusion. The methods used in this paper afford no means of computing $N(m, k)$ when $k$ is small in comparison with $m$, although crude estimates in such a case can be obtained by Lemma $1 .^{8}$

Similarly, the application of the notions developed here to the problem of determining the dimension of $P(m, r, s)$ is limited to the very special cases $r=1$ or $s=m-1$.

\section{BIBLIOGRAPHY}

1. B. Dushnik and E. W. Miller, Partially ordered sets, Amer. J. Math. vol. 63 (1941) pp. 600-610.

2. P. Erdös and G. Szekeres, A combinatorial problem in geometry, Compositio Math. vol. 2 (1935) pp. 463-470.

3. H. Komm, On the dimension of partially ordered sets, Amer. J. Math. vol. 70 (1948) pp. 507-520.

4. E. Szpilrajn, Sur l'extension de l'ordre partiel, Fund. Math. vol. 16 (1930) pp. 386-389.

\section{UNIVERSITY OF MICHIGAN}

${ }^{8}$ The argument in the proof of theorem 4.22 in $[1$, p. 605] shows that $\operatorname{dim} P(m, 1,2)$ $\rightarrow \infty$ as $m \rightarrow \infty$. By Theorem III of this paper we therefore have $\lim _{m \rightarrow \infty} N(m, 3)=\infty$, and by Lemma 1 we conclude that, for fixed $k \geqq 3, \lim _{m \rightarrow \infty} N(m, k)=\infty$, 